Bundesgesundheitsbl - Gesundheitsforsch Gesundheitsschutz 2007 · 50:677-685 DOI 10.1007/s00103-007-0229-3

c) Springer Medizin Verlag 2007

\author{
H. Kahl · A. Schaffrath Rosario · M. Schlaud \\ Robert Koch-Institut, Berlin, BRD
}

\title{
Sexuelle Reifung von Kindern und Jugendlichen in Deutschland
}

\author{
Ergebnisse des Kinder- und Jugendgesund- \\ heitssurveys (KiGGS)
}

\section{Hintergrund}

Die Veränderungen in der körperlichen Entwicklung einschließlich der Reifeentwicklung der Kinder und Jugendlichen im 20. Jahrhundert lassen sich daran erkennen, dass Kinder und Jugendliche heute im Durchschnitt größer und schwerer als ihre Altersgenossen vor 100 Jahren sind und ihre Pubertät früher erleben $[1,2,3$, $4,5,6]$. Während genetische und psychosoziale Faktoren vor allem die individuelle Variabilität in der Entwicklung bestimmen, werden für den säkularen Trend in der Reifeentwicklung Einflüsse einer veränderten Ernährung und eine verbesserte gesundheitliche Betreuung verantwortlich gemacht $[1,7,8,9,10,11]$. Gegenwärtig werden nationale und internationale Diskussionen über mögliche Ursachen eines früheren Pubertätsbeginns insbesondere im Zusammenhang mit der ansteigenden Adipositas im Kindes- und Jugendalter geführt $[12,13,14,15]$. Damit sind auch methodische Fragen zur Bestimmung des Pubertätsbeginns stärker in den Blickpunkt gerückt. Das bezieht sich vor allem auf unterschiedliche Erfassungs- und Auswertungsmethoden der Reifemerkmale bzw. auf das untersuchte Altersspektrum der Stichproben $[16,17]$.

\section{Datenlage}

In den vergangenen 100 Jahren ist das mittlere Alter der Menarche (erste Regelblutung) um ca. 2 Jahre gesunken und hat sich in den 7oer- und 8oer-Jahren des 20. Jahrhunderts auf etwa 12,5-13 Jahre eingependelt [18, 19, 20, 21, 22, 23]. Dieser Trend in der Veränderung des Menarchealters wird durch Untersuchungen sowohl für Deutschland als auch für andere europäische Länder bestätigt [2, 6, 17, 19, 24, 25, 26, 27]. Ergebnisse der 1985 in den jetzigen neuen Bundesländern durchgeführten repräsentativen Reifestudie an ca. 18.00o Kindern und Jugendlichen im Alter von 8-16 Jahren zeigen keinen früheren Reifebeginn (Mädchen: Brustentwicklung (B2) 10,9 Jahre, Menarche 12,7 Jahre; Jungen: Genitalentwicklung (G2) 11,1 Jahre, Spermarche 13,8 Jahre [28, 29, 30, 31]). Im Bericht zur gesundheitlichen Situation von Frauen in Deutschland 2001 wird auf ein derzeitiges Menarchealter von 12,9 Jahren verwiesen [32, S. 281]. Befragungsergebnisse von 14- bis 17-jährigen Jugendlichen im Rahmen der BZgA-Studie zur Körper- und Sexualentwicklung (alte Bundesländer) weisen allerdings auf eine weiter fortschreitende Vorverlegung des Reifealters im Zeitraum von 1981-1994 hin [33]. Danach ist das
Menarchealter von 13,5 auf 12,2 Jahre und das Spermarchealter von 14,2 Jahre auf 12,5 Jahre gesunken. Ergebnisse der aktuellsten Studie der BZgA von 2006 zeigen, dass der Prozentsatz der Mädchen, die ihre erste Regelblutung mit 11-12 Jahren angeben, weiter angestiegen ist [34]. Ein weiterer Gesichtspunkt neben der Veränderung im Menarchealter ist ein Ausgleich der Unterschiede im Menarchealter zwischen sozialen Gruppen. Unterschiede im Menarchealter zwischen Mädchen aus ländlichen Regionen und Großstädten und zwischen Mädchen aus Familien mit unterschiedlichem Sozialstatus waren um die Wende zum 20. Jahrhundert bis in die Mitte des Jahrhunderts deutlich nachweisbar und sind jetzt weniger auffällig $[21,35,36]$.

Dagegen bestehen ethnische Unterschiede nach wie vor. In Bezug auf einen früheren Reifebeginn sind sie für afroamerikanische Mädchen gegenüber weißen amerikanischen Mädchen beschrieben $[37,38,39,40]$, ebenso für in den Niederlanden und in Deutschland lebende türkische Mädchen der 2. und 3. Generation gegenüber einheimischen Mädchen [6, 20, 41, 42]. Angaben aus den schulärztlichen Schulabgangsuntersuchungen in Berlin zeigen von $1980 \mathrm{zu}$ 1995 eine geringe Veränderung im Menar- 
chealter bei deutschen Mädchen von 12,8 auf 12,6 Jahre, bei ausländischen Mädchen von 12,7 auf 12,5 Jahre [43].

\section{Ziel und Fragestellungen}

Kenntnisse über Veränderungen in der Reifeentwicklung der Jugendlichen sind wichtig für die ärztliche Arbeit, aber auch für Erzieher, Lehrer und Eltern. Der Zeitpunkt, zu dem die Reifeentwicklung beginnt und wie sie verläuft, bestimmt auch die Erfordernisse im Hinblick auf Sexualerziehung und Aufklärung und ihre Ausrichtung auf bestimmte Zielgruppen. Die Notwendigkeit der Erarbeitung aktueller und repräsentativer Aussagen zur Reifeentwicklung ergab sich sowohl aus der unzureichenden Datenlage in Deutschland als auch aus der Möglichkeit, Zusammenhänge zwischen dem Reifestatus und anderen sozialen und gesundheitlichen Parametern aus dem Survey abzuklären. Von Interesse war die Klärung der Frage, ob es in der Reifeentwicklung einen Stillstand oder ein Fortschreiten der Akzeleration gibt und inwieweit sich Unterschiede in der Reifeentwicklung nach Region [Ost (inklusive Berlin)/West], Wohnortgröße (ländliche Region = weniger als 5000 Einwohner; kleinstädtische Region $=5000$ bis unter 20.000 Einwohner; mittelstädtische Region $=20.000$ bis unter 100.00o Einwohner, großstädtische Region $=100.000$ Einwohner), Sozialstatus (niedrig, mittel, hoch), Migrationshintergrund (Migrant/Nicht-Migrant) bzw. Herkunftsland (türkisch, russlanddeutsch, sonstige, Nicht-Migrant) und in Abhängigkeit vom Body-Mass-Index (BMI) darstellen.

\section{Teilnehmer und Methoden}

Der Kinder- und Jugendgesundheitssurvey (KiGGS) wurde von Mai 2003 bis Mai 2006 durch das Robert Koch-Institut (RKI) durchgeführt. Konzept, Design und Durchführung der KiGGS-Studie werden in den ersten 6 Beiträgen in diesem Heft ausführlich beschrieben [44, 45, 46, 47, $48,49,50]$. Ziel dieses bundesweiten Befragungs- und Untersuchungssurveys war es, erstmals umfassende und bundesweit repräsentative Daten zum Gesundheitszustand von Kindern und Jugendlichen im Alter von 0-17 Jahren zu erheben. An der Studie nahmen insgesamt 17.641 Kinder und Jugendliche (8656 Mädchen und 8985 Jungen) aus 167 für die Bundesrepublik repräsentativen Städten und Gemeinden teil. Die Teilnahmequote betrug 66,6\%.

Die Reifemerkmale Menarche und Mutation wurden mit der Status-quo-Methode durch die jeweils in den Feldteams tätigen Ärzte erfragt und die Schambehaarung (Pubes) mittels Selbsteinschätzung durch die 10- bis 17-jährigen Jugendlichen erfasst. Von 3803 befragten Mädchen liegen $n=3776$ (99,3\%) Angaben zum Eintritt der Regelblutung (Menarche) vor und $\mathrm{n}=3739$ (98,3\%) Angaben zur Schambehaarung. Von 4028 Jungen antworteten $\mathrm{n}=3950$ (98,1\%), ob und welche Veränderungen in der Stimmlage bemerkt wurden (Stufe 1: Stimme schwankt; Stufe 2: Stimme tief), n=3932 (97,6\%) machten Angaben zur Schambehaarung. Die Selbsteinschätzung der Schambehaarung (Pubes) erfolgte nach Zeichenvorlagen der einzelnen Stufen (Pubic Hair: PH) nach Tanner mit einer Beschreibung [PH1: keine Behaarung (präpuberal), $\mathrm{PH} 2$ : wenige, leicht gekräuselte Haare an Peniswurzel bzw. großen Labien, $\mathrm{PH}_{3}$ : dunklere, gekräuselte Haare über der Symphyse, $\mathrm{PH}_{4}$ : dichteres Haarfeld, Dreieckform, $\mathrm{PH}_{5}$ : sehr dichtes Haarfeld, PH6: weitere Ausdehnung auf die Oberschenkelinnenseite und zum Nabel] $[51,52]$. Die Stufe PH6 wird auch im Erwachsenenalter nicht von allen Personen erreicht, sondern ist abhängig vom individuellen Behaarungstyp. Die Methode der Selbsteinschätzung der Schambehaarung hat zu einer weit höheren Beteiligungsrate bei der Erfassung dieses ausgewählten sekundären Geschlechtsmerkmales geführt als die im Pretest durch eine Ärztin erbetene körperliche Inspektion der Schamregion, die von vielen Kindern und Jugendlichen abgelehnt wurde und sich als besonders problematisch bei den ausländischen Mädchen erwies. Eine Validierungsstudie zur Selbsteinschätzung ist zurzeit noch nicht abgeschlossen.

\section{Statistik}

Um repräsentative Aussagen treffen zu können, wurden die Analysen mit einem
Gewichtungsfaktor durchgeführt, der Abweichungen der Netto-Stichprobe von der Bevölkerungsstruktur (Stand: 31.12. 2004) hinsichtlich Alter (in Jahren), Geschlecht, Region (Ost/West/Berlin) und Staatsangehörigkeit korrigiert. Das mittlere Alter bei Menarche, Mutation und dem Erreichen der verschiedenen Stufen der Schambehaarung wurde über eine Logit-Analyse bestimmt, d.h. durch eine logistische Regression mit dem exakten Lebensalter als stetiger Einflussvariable und dem jeweiligen Ereignis als Zielvariable. Die Konfidenzintervalle für das mittlere Alter wurden dabei auf Grundlage des Fieller-Theorems berechnet. Für die beiden letzten Stufen der Schambehaarung ( $\mathrm{PH}_{5}$ und $\mathrm{PH} 6$ ) lieferte dieses Modell keine gute Beschreibung der Daten und wurde daher ergänzt um das logarithmierte Lebensalter als zusätzliche Einflussvariable.

Konfidenzintervalle und p-Werte wurden mit der Prozedur SURVEYLOGISTIC von SAS 9.1 bestimmt, um die Art der Stichprobenziehung und die daraus resultierende Korrelation der Probanden innerhalb einer Gemeinde zu berücksichtigen. Gruppenunterschiede mit p-Werten von kleiner als 0,05 werden als statistisch signifikant gewertet.

\section{Ergebnisse}

\section{Menarche (erste Regelblutung) und} Mutation (Stimmbruch)

Der Eintritt der Menarche zeigt einen steilen Anstieg von 2,2 \% bei 10-jährigen Mädchen auf 90,7 \% bei 14-jährigen Mädchen. Danach erfolgt bis zum 18. Lebensjahr nur noch eine geringe Zunahme auf 99,3\%. Bei einem großen Anteil der 11-, 12- und 13-jährigen Mädchen ist die Regelblutung noch unregelmäßig, der Anteil verringert sich bis zum 18. Lebensjahr auf $13,2 \%$. Bei den Jungen ist der Eintritt des Stimmbruchs ein relativ spätes Reifemerkmal. Mit 10 Jahren zeigen sich erst bei 0,2\% der Jungen erste Veränderungen in der Stimmlage, mit 14 Jahren haben 47,3\% eine schwankende Stimme und $31,2 \%$ eine tiefe Stimmlage, deren Anteil bis zum 17. Lebensjahr auf 94,4\% steigt. Bei 5,5\% der Jungen ist der Stimmbruch bis zum 18. Lebensjahr noch nicht abgeschlossen, 


\section{Zusammenfassung $\cdot$ Abstract}

Bundesgesundheitsbl - Gesundheitsforsch - Gesundheitsschutz 2007 · 50:677-685

DOI 10.1007/s00103-007-0229-3

(C) Springer Medizin Verlag 2007

\section{H. Kahl · A. Schaffrath Rosario · M. Schlaud}

\section{Sexuelle Reifung von Kindern und Jugendlichen in Deutschland. Ergebnisse des Kinder- und Jugendgesundheitssurveys (KiGGS)}

\section{Zusammenfassung}

Nach einem Stillstand in der Reifeakze-

leration in den 80er-Jahren des 20. Jahr-

hunderts wird international und auch in

Deutschland eine weitere Verschiebung der Reifeentwicklung in das jüngere Lebensalter diskutiert. Mit der Erhebung von Reifemerkmalen bei Mädchen und Jungen im Rahmen des bundesweiten Kinderund Jugendgesundheitssurveys (KiGGS) sollen bevölkerungsrepräsentative Angaben zur sexuellen Reifung ermittelt und Zusammenhänge zwischen Reifestatus und ausgewählten Gesundheits- und Sozialdaten geprüft werden. Mädchen wurden nach der ersten Regelblutung (Menarche) und Jungen nach Veränderungen in der Stimmlage, dem Stimmbruch (Mutation) gefragt (Status-quo-Methode). Die Schambehaarung (Pubes) wurde nach definierten Entwicklungsstufen (Tanner) an- hand von Zeichenvorlagen von den Kindern und Jugendlichen vom vollendeten 10.-17. Lebensjahr selbst eingeschätzt. Das mittlere Alter (Median) für die Menarche, für die Mutation und die Pubesstufen wurde über ein Logit-Modell berechnet. Mit 10 Jahren berichten 42,4\% der Mädchen und 35,7\% der Jungen über die Entwicklung von Schamhaaren. Mit 17 Jahren haben die Mehrzahl der Mädchen und Jungen die Stufen PH5 (Mädchen 57,5\%, Jungen 47,8\%) und PH6 (Mädchen 23,6\%, Jungen 46,5\%) nach Tanner erreicht. Das Durchschnittsalter für die einzelnen Pubesstufen ist bei Mädchen niedriger ( $\mathrm{PH} 2$ 10,8; PH3 11,7; PH4 12,3; PH5 13,4 Jahre) als bei Jungen (PH2 10,9; PH3 12,6; PH4 13,4; PH5 14,1). Der Menarchemedian beträgt 12,8 Jahre, der Median für die Mutation (Stimme tief) 15,1 Jahre. Signifikante
Unterschiede im Menarchealter bestehen zwischen Mädchen in Abhängigkeit vom Sozialstatus (12,7/12,9/13,0 Jahre für niedrigen/mittleren/hohen Sozialstatus) und zwischen Mädchen mit und ohne Migrationshintergrund (12,5/12,9 Jahre). Keine Unterschiede sind im Menarchealter nach Ost/West und nach Wohnortgröße nachweisbar. Zusammenhänge zwischen Reifestatus und BMI sind bei Mädchen stärker ausgeprägt als bei Jungen. Insgesamt beginnt die Reifeentwicklung deutscher Kinder und Jugendlicher im Vergleich zu anderen europäischen Studien nicht signifikant früher.

\section{Schlüsselwörter}

Gesundheitssurvey · Kinder · Jugendliche Sexuelle Reife · Menarche · Mutation · Pubes

\section{Sexual maturation of children and adolescents in Germany. Results of the German Health Interview and Examination Survey for Children and Adolescents (KiGGS)}

\section{Abstract}

Following the standstill in maturity acceleration in the eighties of the twentieth century, now a further shift in maturity development towards younger ages is the issue of an international and also German discussion. The collection of sexual maturity data in boys and girls as part of the nationwide German Health Interview and Examination Survey for Children and Adolescents (KiGGS) is intended to provide population-representative information on sexual maturation and to evaluate associations between maturity status and selected health and social data. Girls were interviewed regarding their first menstrual period (menarche) and boys regarding voice change (status-quo method). Pubic hair was self-assessed by children and ado- lescents from 10 to 17 years of age, based on drawings of Tanner's defined developmental stages. The median age for menarche, for voice change and pubic hair stages were calculated using a logit model. At an age of 10 years, $42.4 \%$ of girls and $35.7 \%$ of boys report the development of pubic hair. At 17 years of age, the majority of girls and boys have reached the stages $\mathrm{PH} 5$ (girls $57.5 \%$, boys $47.8 \%$ ) and PH6 (girls $23.6 \%$, boys $46.5 \%$ ) according to Tanner. The average age for each pubic hair stage is lower in girls (PH2 10.8; PH3 11.7; PH4 12.3; $\mathrm{PH} 513.4$ years) than in boys ( $\mathrm{PH} 2$ 10.9; PH3 12.6; PH4 13.4; PH5 14.1). The median age at menarche is 12.8 years, the median for voice change (voice low) 15.1 years. Significant differences in age at me- narche are found in girls depending on socioeconomic status (12.7/12.9/13.0 years for low/middle/high status) and between girls with and without migration background ( $12.5 / 12.9$ years). No differences in age at menarche can be seen between East and West Germany or cities and rural areas. The association between maturity status and BMI is more pronounced in girls than in boys. Overall, the onset of maturity development in German children and adolescents is not significantly earlier than in other European studies.

\section{Keywords}

Health survey - Children · Adolescents . Sexual maturity · Menarche $\cdot$ Mutation . Pubic hair 
darunter ist bei $1,4 \%$ sogar noch eine kindliche Stimmlage vorhanden.

Die - Abb. 1 zeigt einen Menarchemedian von 12,8 Jahren für die Mädchen und Medianwerte für die Mutation Stufe 1 (Stimme schwankt) von 13,5 Jahren und für die Stufe 2 (Stimme tief) von 15,1 Jahren für die Jungen. Der Zeitraum vom Beginn der Mutation (schwankende Stimme) bis zur Ausprägung der tiefen Stimmlage beträgt ca. 1,5 Jahre.

\section{Pubes (Schambehaarung)}

Die Grafiken in $\bullet$ Abb. 2 und $\bullet$ Abb. 3 verdeutlichen eine kontinuierliche Abfolge der Entwicklungsstufen der Schambehaarung bei Jungen und Mädchen.

Die raschere Entwicklung der Schambehaarung bei den Mädchen gegenüber den Jungen wird erst ab der Pubesstufe $\mathrm{PH}_{3}$ deutlich, denn mit 10 Jahren erreichen nach der Selbsteinschätzung sowohl Jungen mit 31,9\% und Mädchen mit 29,3\% $\mathrm{zu}$ je einem Drittel die Stufe PH2. 13,1\% der Mädchen geben aber bereits die höheren Ausprägungsstufen $\mathrm{PH}_{3}-\mathrm{PH}_{5}$ der Schambehaarung an, Jungen nur zu 3,8 \%. Die Tatsache, dass bei 13-jährigen Mädchen und bei Jungen mit 13 und 14 Jahren alle Entwicklungsstufen der Schambehaarung vorhanden sind, unterstreicht die große Varianz in der Reifeentwicklung. Im Alter von 17 Jahren haben $57,5 \%$ der Mädchen die Stufe $\mathrm{PH}_{5}$ als damit häufigster Ausprägungsform der Schambehaarung bei Mädchen erreicht, 23,6\% geben die Stufe PH6 an, 18,1\% verbleiben in der Stufe $\mathrm{PH}_{4}$. Bei 17-jährigen Jungen weisen $47,7 \%$ der Jungen die Stufe $\mathrm{PH}_{5}$ auf, 46,5\% die Stufe PH6. Der höhere Anteil von Jungen in der Pubesstufe PH6 entspricht dem geschlechtstypischen männlichen Behaarungstyp.

Die Darstellung des jeweiligen medianen Alters für die erreichten Stufen der Schambehaarung (• Tabelle 1 ) zeigt einen fast zeitgleichen Beginn des Wachstums der Schamhaare mit 10,8 Jahren bei Mädchen und mit 10,9 Jahren bei Jungen. Inwieweit hier methodische Aspekte durch die Selbsteinschätzung des Merkmals eine Rolle spielen können, muss noch überprüft werden. Für die späteren Stufen ist der Entwicklungsvorsprung der Mädchen deutlich am jeweiligen nied-

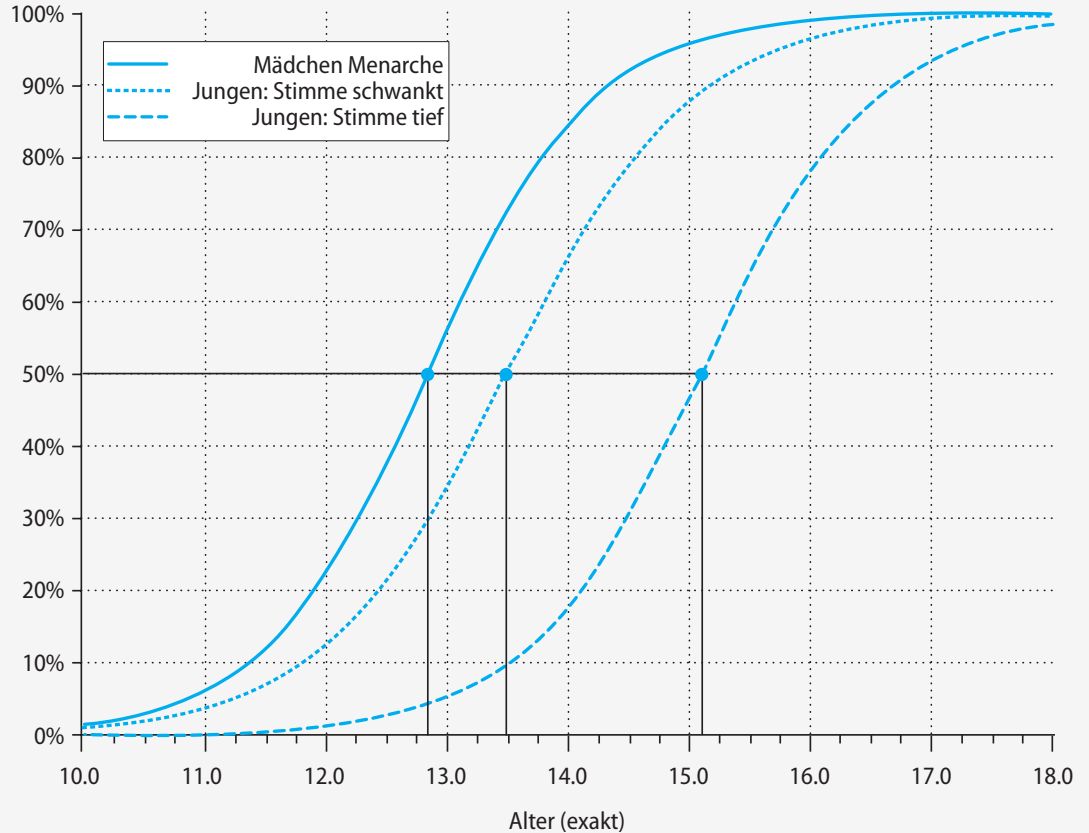

Abb. $1 \Delta$ Mittleres Alter in Jahren (Median gemäß Logit-Analyse) für Menarche (erste Regelblutung) und Mutation (Stimmbruch: Stufe 1 = Stimme schwankt, Stufe 2 = Stimme tief)

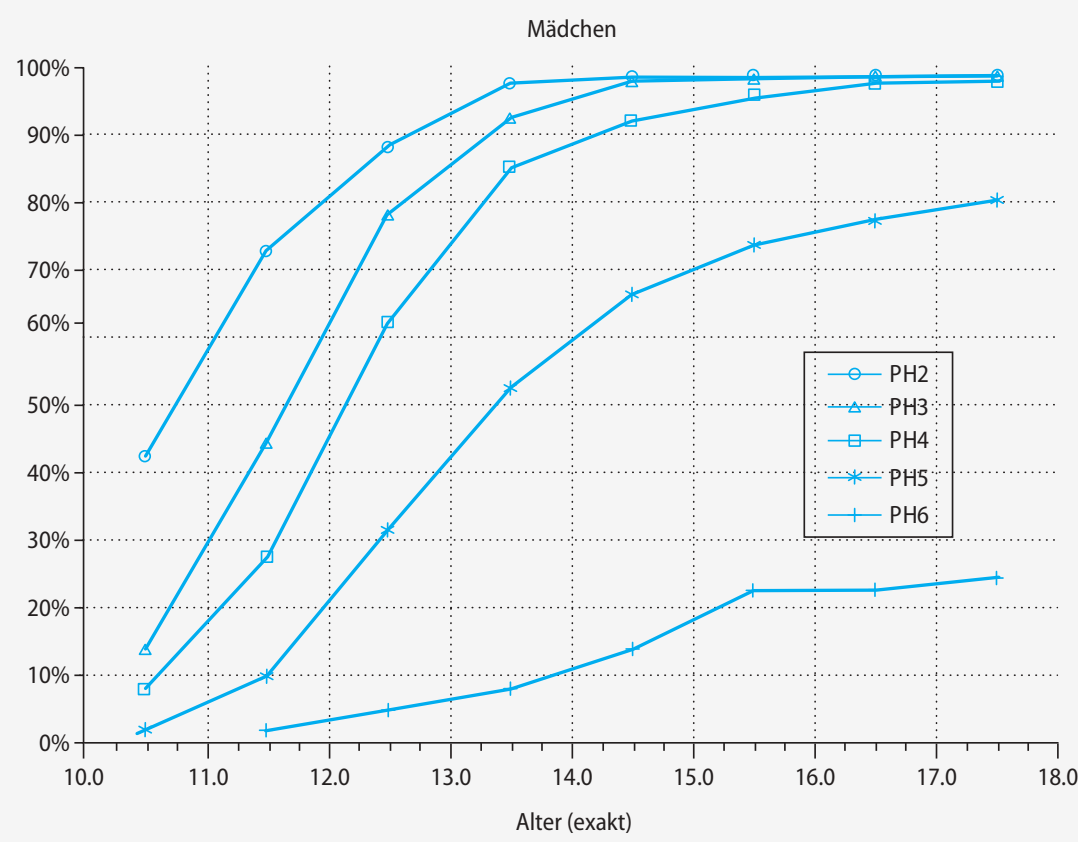

Abb. $2 \Delta$ Stufen der Schambehaarung (Pubes) bei Mädchen nach Alter in \%, Selbsteinschätzung nach Zeichenvorlage (Tanner)

rigeren mittleren Alter bei den einzelnen Pubesstufen ablesbar. Der Eintritt der Menarche bei Mädchen ist im Durchschnitt nach Erreichen der Entwicklungsstufen $\mathrm{PH}_{4}$ wahrscheinlich. Bei Jungen fällt der Beginn des Stimmbruchs (schwankende
Stimme) in etwa mit dem Erreichen der Pubesstufe $\mathrm{PH}_{4}$ zusammen. Eine tiefe Stimmlage tritt in der Regel erst nach Erreichen der Pubesstufe $\mathrm{PH}_{5}$ auf. 


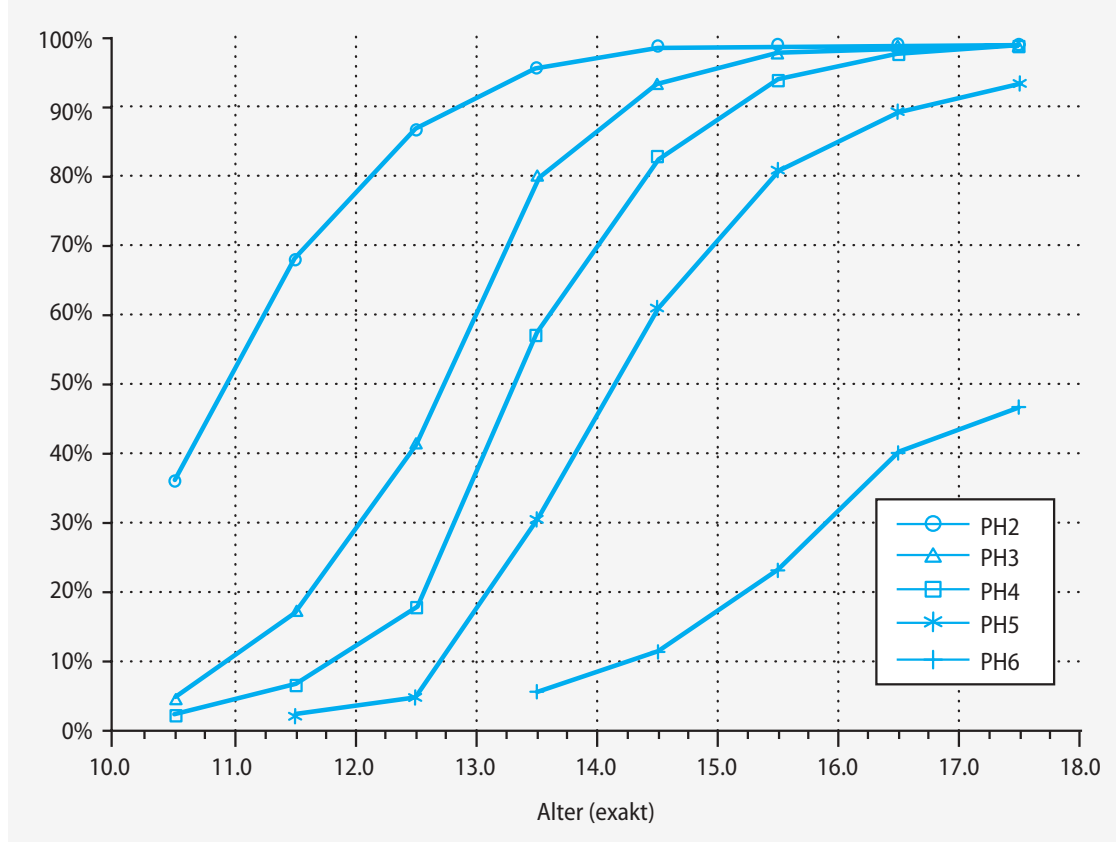

Abb. $3 \Delta$ Stufen der Schambehaarung bei Jungen nach Alter in \%, Selbsteinschätzung nach Zeichenvorlage (Tanner)

\section{Tabelle 1}

Mittleres Alter in Jahren (Median gemäß Logit-Analyse) für die Stufen der Schambehaarung (Pubesbehaarung: PH), die Menarche (erste Regelblutung) und die Mutation (Stimmbruch: Stufe 1 = Stimme schwankt, Stufe 2 = Stimme tief)

\begin{tabular}{|c|c|c|c|c|c|c|}
\hline & \multicolumn{5}{|c|}{$\begin{array}{l}\text { Reifemerkmale } \\
\text { mittleres Alter (Jahre) und } 95 \% \text {-Konfidenzintervalle }\end{array}$} & \multirow[b]{2}{*}{ Mutation } \\
\hline & PH2 & PH3 & PH4 & PH5 ${ }^{a}$ & Menarche & \\
\hline Mädchen & $\begin{array}{l}10,8 \\
(10,6-10,9)\end{array}$ & $\begin{array}{l}11,7 \\
(11,6-11,8)\end{array}$ & $\begin{array}{l}12,3 \\
(12,2-12,3)\end{array}$ & 13,4 & $\begin{array}{l}12,8 \\
(12,8-12,9)\end{array}$ & \\
\hline Jungen & $\begin{array}{l}10,9 \\
(10,8-11,1)\end{array}$ & $\begin{array}{l}12,6 \\
(12,6-12,7)\end{array}$ & $\begin{array}{l}13,4 \\
(13,3-13,5)\end{array}$ & 14,1 & & $\begin{array}{l}\text { St. } 113,5 \\
(13,4-13,6) \\
\text { St. } 215,1 \\
(15,0-15,2)\end{array}$ \\
\hline
\end{tabular}

\section{Soziale Einflussfaktoren}

Ausgehend von der These des umweltbedingten Einflusses auf die Reifeentwicklung, wird untersucht, inwieweit sich gruppenspezifische Unterschiede in der Reifentwicklung zwischen Kindern und Jugendlichen nach Region (Ost/West), Sozialstatus, Migrationsstatus bzw. Herkunftsland und in Abhängigkeit vom Body-Mass-Index (BMI) nachweisen lassen (- Tabelle 2).

Die Auswertung der Reifeentwicklung nach Ost/West und nach Wohnortgröße zeigt sowohl bei Mädchen als auch bei Jungen keine statistisch signifikanten Unterschiede. Dagegen sind signifikante Unterschiede im Menarchealter bei Mädchen in Abhängigkeit vom Sozialstatus nachweisbar (12,7 über 12,9 zu 13, O Jahren bei Mädchen aus Familien mit niedrigem, mittlerem bzw. hohem Sozialstatus). Bei Jungen sind die Unterschiede im mittleren Mutationsalter nicht statistisch signifikant.

Die Auswertung nach dem Migrati- onsstatus zeigt beim Menarchealter ein statistisch signifikant früheres Menarchealter bei Mädchen mit einem Migrationshintergrund im Vergleich zu deutschen Mädchen (12,5 vs. 12,9 Jahre). Bei den Jungen sind keine Unterschiede im mittleren Mutationsalter ausgeprägt. Da der Migrationsstatus Kinder und Jugendliche aus unterschiedlichen Herkunftsländern zusammenfasst, erscheint eine Differenzierung der Daten nach der Herkunft sinnvoll. Dabei unterscheidet sich das Menarchealter zwischen Mädchen türkischer Herkunft (12,5 Jahre), russlanddeutschen Mädchen (12,4 Jahre) und Mädchen aus anderen Herkunftsländern (12,5 Jahre) kaum. Bei Jungen aus unterschiedlichen Herkunftsländern sind die Unterschiede im mittleren Mutationsalter nicht statistisch signifikant.

\section{Zusammenhang zwischen Reife und BMI}

Zwischen dem Menarchealter und dem BMI bestehen bei den Mädchen signifikante Zusammenhänge (• Tabelle 3). Das mittlere Menarchealter beträgt bei adipösen Mädchen 12,1 Jahre, bei stark untergewichtigen Mädchen dagegen 14,9 Jahre, das bedeutet einen Unterschied von 2,8 Jahren. Deutliche Unterschiede bestehen diesbezüglich auch im Hinblick auf das mediane Alter für die verschiedenen Stufen der Schambehaarung. Mädchen mit einem höheren BMI beginnen ihre Entwicklung früher und beenden sie früher. Bei den Jungen ist die Differenz bezüglich des mittleren Alters der Stufen der Schambehaarung in Abhängigkeit vom BMI weniger stark ausgeprägt, aber ebenfalls signifikant. Zwischen dem mittleren Mutationsalter der adipösen und der stark untergewichtigen Jungen liegt ein Abstand von o,7 Jahren.

\section{Diskussion}

Das Fehlen vergleichbarer repräsentativer Daten erschwert die Einschätzung eines Trends in der Reifeentwicklung der Kinder und Jugendlichen in Deutschland. Der Survey ergab ein mittleres Menarchealter von 12,8 Jahren und ein mittleres Mutationsalter von 13,5 Jahren (Stufe 1) bzw. 15,1 Jahren (Stufe 2). Legt man das 1985 in 
Tabelle 2

Mittleres Alter (Median gemäß Logit-Analyse) für Menarche (erste Regelblutung), Mutation (Stimmbruch Stufe 2=Stimme tief) nach Ost/West, Sozialstatus, Wohnortgröße, Migrationsstatus und Herkunftsland in Jahren

\begin{tabular}{|c|c|c|c|c|c|c|}
\hline \multirow[t]{2}{*}{ Variable } & \multicolumn{3}{|c|}{ Mädchen } & \multicolumn{3}{|c|}{ Jungen } \\
\hline & $\mathbf{n}$ & Menarche & $\mathbf{p}$ & n & $\begin{array}{l}\text { Mutation } \\
\text { (Stufe 2) }\end{array}$ & $\mathbf{p}$ \\
\hline Gesamt & 3776 & 12,8 & & 3956 & 15,1 & \\
\hline Ost & 1278 & 12,8 & & 1290 & 15,0 & \\
\hline West & 2498 & 12,9 & $p=0,28$ & 2666 & 15,1 & $p=0,66$ \\
\hline \multicolumn{7}{|c|}{$\begin{array}{l}\text { Wohnortgröße } \\
\text { (Einheit Einwohnerzahl) }\end{array}$} \\
\hline$<5000$ & 840 & 12,8 & & 857 & 15,0 & \\
\hline $5000-<20000$ & 967 & 12,9 & & 1038 & 15,1 & \\
\hline $20000-<100000$ & 1111 & 12,9 & & 1114 & 15,2 & \\
\hline 100000 und $>$ & 858 & 12,8 & $p=0,65$ & 947 & 15,0 & $p=0,81$ \\
\hline \multicolumn{7}{|l|}{ Sozialstatus } \\
\hline Niedrig & 985 & 12,7 & & 1043 & 15,2 & \\
\hline Mittel & 1784 & 12,9 & & 1844 & 15,1 & \\
\hline Hoch & 898 & 13,0 & $p=0,01$ & 929 & 15,0 & $p=0,08$ \\
\hline Migrant & 567 & 12,5 & & 635 & 15,0 & \\
\hline Nicht-Migrant & 3203 & 12,9 & $p<0,001$ & 3318 & 15,1 & $p=0,25$ \\
\hline \multicolumn{7}{|l|}{ Herkunftsland } \\
\hline Türkisch & 132 & 12,5 & & 171 & 15,0 & \\
\hline Russlanddeutsch & 178 & 12,4 & & 182 & 15,1 & \\
\hline Sonstige Herkunft & 257 & 12,5 & & 282 & 14,9 & \\
\hline Nicht-Migrant & 3203 & 12,9 & $p<0,001$ & 3318 & 15,1 & $p=0,39$ \\
\hline
\end{tabular}

der Reifestudie der jetzt neuen Bundesländer ermittelte Menarchealter von 12,7 Jahren [31] zugrunde, ergibt sich im Vergleich zum jetzt ermittelten Menarchealter für die Region Ost von 12,8 Jahren zumindest für die neuen Bundesländer diesbezüglich kein weiteres Absinken. Dagegen wurde für die alten Bundesländer nach den Ergebnissen der BZgA-Studie im Zeitraum von 1980-1994 ein Absinken des Menarchealters von 12,7 auf 12,2 Jahre errechnet. Danach würde das im Survey ermittelte Menarchealter von 12,9 Jahren für die Region West eine Erhöhung bedeuten. Es bleibt zu klären, inwieweit methodische Aspekte des Untersuchungsdesigns und des zugrunde liegenden Altersspektrums in den beiden Studien für diese unterschiedlichen Ergebnisse von Bedeutung sind. Während die Ergebnisse der BZgAStudie auf retrospektiven Angaben 14- bis 17-jähriger Jugendlicher zur Menarche und zur Spermarche beruhen, wurde im Survey die Status-quo-Methode angewendet, die als zuverlässiger in der Literatur beschrieben ist $[33,53]$.
Das im Survey ermittelte mediane Menarchealter von 12,8 Jahren bewegt sich durchaus im Rahmen der aus anderen europäischen Studien mitgeteilten Ergebnisse. Danach liegt das Menarchealter seit den 8oer-Jahren des vorigen Jahrhunderts im Bereich zwischen 12,6 und 13,3 Jahre $[2,5,17,18,19]$. Repräsentative Ergebnisse zur Reifeentwicklung bei Jungen, speziell zum Merkmal Mutation, sind in Deutschland kaum publiziert, sodass keine Vergleiche möglich sind. Das ermittelte mittlere Alter für die einzelnen Pubesstufen bei Jungen unterscheidet sich außer für die $\mathrm{PH}_{2}$ kaum von den in der Literatur mitgeteilten Angaben [40, 45]. Das für $\mathrm{PH} 2$ im Survey für Jungen ermittelte mittlere Alter von 10,9 Jahren für das Auftreten der ersten Schamhaare erscheint zu niedrig. Auf diese Überschätzung des Wachstums der Schambehaarung wird in der Literatur aufmerksam gemacht, da besonders jüngere Kindern auch die feine Körperbehaarung in der Selbsteinschätzung bereits als eine Stufe werten [54].
Interessant sind die Zusammenhänge zwischen ausgewählten Einflussfaktoren und dem mittleren Alter bei Menarcheeintritt, Mutation oder für das Erreichen der verschiedenen Pubesstufen. Statistisch signifikante Unterschiede im Reifestatus zeigen sich bei Mädchen nur nach dem Sozialstatus, dem Migrationsstatus bzw. dem Herkunftsland. Dagegen sind Ost-WestUnterschiede und Unterschiede nach Wohnortgröße im Menarchealter nicht mehr nachweisbar. Anscheinend gleichen sich bestimmte Merkmale innerhalb der Regionen an. Ein umgekehrter Trend gegenüber früheren Untersuchungen stellt sich bei den Ergebnissen zum Sozialstatus dar. Danach hatten Mädchen aus Familien mit niedrigem Sozialstatus ihre erste Regelblutung im Durchschnitt später als Mädchen aus Familien mit hohem Sozialstatus. Mit 13,0 Jahren zeigen Mädchen aus Familien mit hohem Sozialstatus jetzt ein höheres mittleres Menarchealter als Mädchen aus Familien mit niedrigem Sozialstatus mit 12,7 Jahren. Inwieweit hier auch die zunehmende Adipositas bei Mädchen aus Familien mit niedrigem Sozialstatus eine Bedeutung hat, bedarf weiterer Klärung. Bei Jungen sind sowohl für die Schambehaarung als auch für die $\mathrm{Mu}-$ tation keine Unterschiede nach den oben genannten Einflussgrößen feststellbar.

Die im KiGGS nachgewiesenen Unterschiede im Menarchealter zwischen türkischen (12,5 Jahre) und deutschen Mädchen (12,9 Jahre) sind in regionalen Studien in Deutschland beschrieben und werden auch aus anderen europäischen Ländern berichtet $[6,20,41,42,43]$. Türkische Mädchen, die in den Niederlanden leben, zeigen auch in der 2.und 3. Generation ein niedrigeres Menarchealter als die holländischen Mädchen (12,8 vs. 13,3 Jahre). Zur Reifeentwicklung von Mädchen mit Migrationshintergrund anderer Herkunft (russlanddeutsche, sonstige Herkunft) sind keine Ergebnisse bekannt. Bei Jungen stellen sich Unterschiede nach Herkunftsland nicht so ausgeprägt dar wie für Mädchen. Für die Praxis sind diese Kenntnisse sowohl für Ärzte als auch Lehrer und Erzieher wichtig.

Seit langem ist bekannt, dass Ernährungsstatus und Pubertätsentwicklung eng miteinander verknüpft sind. So führt ein Mangel an Fettmasse oft zu einer spät 
Tabelle 3

Mittleres Alter in Jahren (Median gemäß Logit-Analyse) für die Stufen der Schambehaarung (Pubesbehaarung, PH), Menarche (erste Regelblutung) und Mutation (Stimmbruch Stufe 2 = Stimme tief) nach Body-Mass-Index (BMI)

\begin{tabular}{|c|c|c|c|c|c|c|c|c|c|c|c|c|}
\hline \multirow[t]{2}{*}{ BMI ${ }^{\mathbf{a}}$} & \multicolumn{6}{|c|}{ Mädchen } & \multicolumn{6}{|c|}{ Jungen } \\
\hline & PH2 & PH3 & PH4 & PH5 & $\mathbf{N}$ (Men) & Menarche & PH2 & PH3 & PH4 & PH5 & N (Mut.) & $\begin{array}{l}\text { Mutatior } \\
\text { (Stufe 2) }\end{array}$ \\
\hline
\end{tabular}

Stark unter-

gewichtig

\begin{tabular}{|c|c|c|c|c|c|c|c|c|c|c|c|c|}
\hline$(<\mathrm{P} 3)$ & 11,2 & 12,5 & 13,6 & 15,5 & 73 & 14,9 & 11,2 & 13,2 & 14,5 & 15,0 & 97 & 15,7 \\
\hline \multicolumn{13}{|l|}{ Untergewicht } \\
\hline$(\mathrm{P} 3-<\mathrm{P} 10)$ & 11,7 & 12,5 & 13,5 & 14,6 & 198 & 14,2 & 11,4 & 12,8 & 13,8 & 14,9 & 215 & 15,5 \\
\hline \multicolumn{13}{|l|}{ Normal } \\
\hline$(>\mathrm{P} 10-<\mathrm{P} 90)$ & 10,8 & 11,7 & 12,2 & 13,5 & 2.811 & 12,9 & 10,9 & 12,6 & 13,4 & 14,1 & 2.929 & 15,1 \\
\hline \multicolumn{13}{|l|}{ Übergewicht } \\
\hline (>P90 < P97) & 10,1 & 11,4 & 11,8 & 13,0 & 379 & 12,2 & 11,0 & 12,6 & 13,2 & 14,0 & 414 & 15,1 \\
\hline \multicolumn{13}{|l|}{ Adipös } \\
\hline (> P97) & 10,4 & 11,1 & 11,6 & 12,6 & 291 & 12,1 & 10,2 & 12,4 & 13,2 & 14,1 & 286 & 15,0 \\
\hline$p$ & $p<0,001$ & $p<0,001$ & $p<0,001$ & $p<0,001$ & & $p<0,001$ & $p=0,022$ & $p=0,034$ & $p<0,001$ & $p=0,01$ & & $p=0,026$ \\
\hline
\end{tabular}

a P3, P10, P90, P97: alters- und geschlechtsspezifische Perzentile nach Kromeyer-Hauschild

einsetzenden Pubertät bzw. einem Ausbleiben der Regelblutung, bekannt z. B. durch Untersuchungen bei Leistungssportlerinnen. Das hat die Annahme unterstützt, dass ein gewisses „kritisches“ Körpergewicht bzw. ein gewisser Anteil an Körperfett (>17\%) für den Eintritt in die Pubertät maßgeblich sein könnte [21]. Ergebnisse aus den USA über eine immer früher einsetzende Brustentwicklung bei Mädchen haben eine erneute Diskussion darüber ausgelöst, ob und inwieweit die zunehmende Adipositas unter den Kindern mit einem früheren Reifebeginn in Zusammenhang steht [12]. Es wird angenommen, dass das Fettgewebe mit der Bildung des hormonähnlichen Eiweißes Leptin ein metabolisches Signal für den Beginn der Reifeentwicklung gibt und damit auch die hormonellen Vorgänge insgesamt beeinflusst werden [10].

Nach den Surveydaten besteht ebenfalls ein signifikanter Zusammenhang zwischen einem frühen Menarchealter, einer frühen Entwicklung der Schambehaarung und einem hohen BMI bei Mädchen, während sich ein solcher bei Jungen weniger deutlich nachweisen lässt. Bei der Diskussion um höhere BMI-Werte und ihre Bedeutung für den Reifebeginn scheinen auch noch andere Faktoren der Körperkonstitution eine Rolle zu spielen. Ein Ergebnis der 1985 durchgeführten
Reifestudie (Ost) zeigt, dass Mädchen mit einem gedrungenen Körperbautyp (Pyknomorphe) im Durchschnitt früher reifen als schlank gebaute Mädchen (Leptomorphe) [31]. In weiteren Untersuchungen wäre zu klären, inwieweit ein erhöhter BMI-Wert eher als Ursache oder womöglich als Folge einer früheren Reife einzustufen ist und welche weiteren Einflussfaktoren dabei eine Rolle spielen. Um auch andere Einflüsse auf einen früheren Reifebeginn zu untersuchen, bieten die im Survey erfassten Körpermesswerte, u. a. die Hautfaltendicke, und andere Angaben wie z. B. zum psychosozialen Umfeld, vielseitige Ansatzpunkte.

\section{Fazit}

Insgesamt schafft der KiGGS-Datensatz erstmalig die Möglichkeit, anhand einer Vielzahl repräsentativer Angaben zu Gesundheits- und Laborparametern viele der im Zusammenhang mit der Reifeentwicklung diskutierten Fragestellungen zu prüfen und neue Erkenntnisse zu erarbeiten. Die methodische Herangehensweise bei der Erfassung und Auswertung von Angaben zur Reifeentwicklung kann einen wesentlichen Einfluss auf die Ergebnisse und darauf aufbauende Schlussfolgerungen zur Reifeakzeleration haben. Aufgrund eigener Erfahrungen und auch im Hinblick auf die in der Literatur hierzu diskutierten Probleme soll auf einige Voraussetzungen bei entsprechenden Untersuchungsvorhaben hingewiesen werden. Im Vordergrund stehen dabei:

- Die Einbeziehung eines ausreichend großen Altersspektrums von 8-18 Jahren für die Erfassung der Reifemerkmale. Diese Schlussfolgerung ergibt sich auch aus dem Ergebnis im Survey, da bereits ca. $40 \%$ der 10 -jährigen Mädchen eine Schambehaarung angaben.

- Die Wahl der Erfassungsmethoden. In Abhängigkeit vom Untersuchungsanliegen einer Studie können Befragungsdaten zur Reifeentwicklung (Menarche, Spermarche bzw. Mutation) eine erste Orientierung über den Entwicklungsstand liefern, wobei Fragen zum Status quo validere Ergebnisse als die retrospektive Erfragung des Menarche-, Spermarche- bzw. Mutationsalters liefern. Werden Aussagen zum durchschnittlichen Beginn und zur Dauer der Reifeentwicklung angestrebt, ist die standardisierte Inspektion/Palpation der sekundären Reifemerkmale durch Ärzte theoretisch die Methode der Wahl. Im Rahmen von Surveys spricht jedoch die größere Teilnahmebereitschaft der Kinder und Jugendlichen beim Einsatz 
der Methode „Selbsteinschätzung“ der Reifemerkmale mehr für deren Anwendung als für die Durchführung einer ärztlichen Inspektion. Bei Anwendung von Verfahren der Selbsteinschätzung der Reifemerkmale sollte die Validität geprüft werden [16], wie dies auch im KiGGS erfolgte.

- Die Verwendung verschiedener statistischer Auswertungsverfahren für die Berechnung des mittleren Alters der ausgewählten Merkmale kann zu unterschiedlichen Ergebnissen führen. Die Methode sollte daher stets beschrieben sein [53].

\section{Korrespondierende Autorin}

\section{Dr. Heidrun Kahl}

Robert Koch-Institut

Abteilung für Epidemiologie und

Gesundheitsberichterstattung

Postfach 650261

13302 Berlin, BRD

E-Mail:kahlh@rki.de

\section{Literatur}

1. Remschmid H (1992) Adoleszenz. Entwicklung und Entwicklungskrisen im Jugendalter, 1. Aufl. Thieme Stuttgart

2. Hauspie RC, Vercauteren M, Susanne C (1997) Secular changes in growth and maturation: an update. Acta Paediatrica Suppl 423:20-27

3. Jaeger U (1998) Secular trend in Germany. In: Bodzsar BE, Susanne C (eds) Secular growth changes in Europe. Eötvös Univ. Press, Budapest, pp 135-159

4. Hesse V, Jaeger U, Vogel H et al. (1997) Wachstumsdaten deutscher Kinder von der Geburt bis zu 18 Jahren. Sozialpädiatrie 19:20-22

5. Cole TJ (2000) Secular trends in growth. Proceed Nutr Society 59:317-324

6. Fredriks AM, van Buuren $S$, Jeurissen SER et al. (2003) Height, weight, body mass index and pubertal development reference values for children of Turkish origin in the Netherlands. Eur J Pediatrics 162:788-793

7. Kracke B, Silbereisen RK (1994) Körperliches Entwicklungstempo und psychosoziale Anpassung im Jugendalter: Ein Überblick zur neueren Forschung. Z Entwicklungspsychol Pädagog Psychol 26: 293-330

8. Alsaker $F$ (1995) Timing of puberty and reactions to pubertal changes. In: Rutter M (Hrsg) Psychosocial disturbances in young people: Challenges for prevention: 37-67. Cambridge University Press, Cambridge

9. Ge X, Conger RD, Elder GH Jr (2001) Pubertal transition, stressful life events and the emergence of gender differences in adolescent depressive symptoms. Development Psychol 37:404-417
10. Silbereisen RK, Kracke B (1997) Self-reported maturational timing and adaptation in adolescence. In: Schulenberg J, Maggs JL, Hurrelmann K (eds) Health risks and developmental transitions during adolescence. New Cambridge University Press, York, pp 85-109

11. Delemarre-van de Waal HA (2005) Secular timing of puberty. Endocr Dev 8:1-14

12. Herman-Giddens ME, Slora E, Wasserman RC et al. (1997) Secondary sexual characteristics and menses in young girls seen in the office practice: a study from the Pediatric Research in Office Settings network. Pediatrics 99:502-512

13. Herman-Giddens ME (2001) Secondary sexual characteristics in boys. NHANES III, 1988-1994. 155:1022-1028

14. Karlberg J (2002) Secular trends in pubertal development. Horm Res 57:19-30

15. Dunger DB (2005) Effects of obesity on growth and puberty. Best Practice Res Clin Endocrinol Metabolism 19:375-390

16. Coleman L, Coleman J (2002) The measurement of puberty: a review. J Adolescence 25:535-550

17. De Munich, Keizer-Schrama SMPF, Mul D (2001) Trends in pubertal development in Europe. Human Reproduction Update 7:287-291

18. Barth H, Döbler T, Amon K (1984) Zur Stagnation der Menarche. Ärztl Jugendk 75:303-307

19. Danker-Hopfe H (1986) Die säkulare Veränderung des Menarchealters in Europa. E. Schweizerbartsche Verlagsbuchhandlung (Nägele und Obermiller), Stuttgart

20. Ostersehlt D, Danker-Hopfe H (1991) Changes in age at menarche in Germany: evidence for a continuing decline. Am J Human Biol 3:647-654

21. Reißig M (1985) Körperliche Entwicklung und Akzeleration Jugendlicher. Ergebnisse einer Längsschnittuntersuchung an Schülern vom 12.-16. Lebensjahr. Verlag Volk und Gesundheit, Berlin

22. Richter J, Wenske HJ (1985) Hat die Akzeleration ein Ende? - „Zur Diskussion gestellt" Ärztl. Fortb 79:255-257

23. Richter J (1989) Ergebnisse langfristiger Entwicklungsbeobachtungen bei Mädchen. Sozialpädiatrie Praxis Klinik 11:650-657

24. Danubio ME, de Simone M, Vecchi F et al. (2004) Age at menarche and age of onset of pubertal charcteristics in 6-14-years-old girls from the Province of L'Aquila (Abruzzo,Italy). Am J Human Biol 16:470-478

25. Lindgren G (1996) Pubertal stages of Stockholm schoolchildren. Acta Paediatr 85: 1365-1367

26. Bodzsar EB (2000) Studies on sexual maturation of Hungarian children. Acta Biologica Szegediensis 44:155-165

27. Juul A, Teilmann G, Scheike T et al. (2006) Pubertal development in Danish children: comparison of recent European and US data. Int J Androl 29: 247-255

28. Kahl H, Richter J, Sommer K (1988) Wachstum und sexuelle Reifung bei Kindern und Jugendlichen in der DDR. Wiss. Zeitschrift der Humboldt-Univ. zu Berlin, R Med 37:185-186

29. Engelhardt L, Willers B, Pelz L (1995) Sexual maturation in East German girls. Acta Paediatr 84: 1362-1365

30. Willers B, Engelhardt L, Pelz L (1996) Sexual maturation in East German boys. Acta Pediatr 85: 785-788

31. Greil H, Kahl H (2005) Assesment of development age: cross-sectional analysis of secondary sexual characteristics. Anthrop Anz 63:63-75
32. Bundesministerium für Familie, Senioren, Frauen und Jugend (2001) Bericht zur gesundheitlichen Situation von Frauen in Deutschland. Eine Bestandsaufnahme unter Berücksichtigung der unterschiedlichen Entwicklung in West- und Ostdeutschland, 1. Aufl. Kohlhammer, Berlin, S 28

33. Kluge N (1998) Sexualverhalten Jugendlicher heute: Ergebnisse einer repräsentativen Jugend- und Elternstudie über Verhalten und Einstellungen zur Sexualität. Juventa, Weinheim München

34. Bundeszentrale für gesundheitliche Aufklärung (2002) Jugendsexualität. Wiederholungsbefragung von 14- bis 17-Jährigen und ihren Eltern. Ergebnisse der Repräsentativbefragung. Eigenverlag, Köln

35. Ahrendt H-J (1985) Geschlechtliche Entwicklung, Sexualverhalten und Kontrazeption 15- bis 17jähriger weiblicher Jugendlicher. Medizinische Akademie, Magdeburg; Med. Habilitation

36. Fritsche U (1993) Frau '90 - Berliner Studie zum reproduktiven Verhalten der Frauen und Paare und seine sozialökonomischen und psychosozialen Determinanten. Senatsverwaltung für Frauen und Arbeit, Berlin

37. Cash TF, Henry PE (1995) Women's body image: The results of a national survey in the USA. Sex Roles 33:19-28

38. Sun SS, Schubert CM, Chumlea WS et al. (2002) National estimates of the timing of sexual maturation and racial differences among US children. Pediatrics 110:911-919

39. Chumlea WC, Schubert CM, Roche AF et al. (2003) Age et menarche and racial comparisons in US girls. Pediatrics 111:110-113

40. Wu T, Mendola P, Buck GM (2002) Ethic differences in the presence of secondary sex characteristics and menarche among US Girls: the third national health and nutrition examination survey, 1988-1994. Pediatrics 110:752-757

41. Zabransky S, Weinand C, Schmidgen A et al. (2000) Saarländische Wachstumsstudie 1995. Kinder Jugendarzt 31:822-827

42. Georgi M, Schaefer F, Wühl E, Schärer K (1996) Körpergröße und -gewicht bei gesunden Schulkindern und Jugendlichen in Heidelberg. Monatsschr. Kinderheilkd 144:813-824

43. Kiss A (1997) Gesundheit und Gesundheitsverhalten von Jugendlichen in Berlin unter besonderer Berücksichtigung der Ergebnisse der Schulentlassungsuntersuchungen 1994/95. Senatsverwaltung für Gesundheit und Soziales, Berlin

44. Kurth B-M (2007) Der Kinder- und Jugendgesundheitssurvey (KiGGS): Ein Überblick über Planung, Durchführung und Ergebnisse unter Berücksichtigung von Aspekten eines Qualitätsmanagements. Bundesgesundheitsbl Gesundheitsforsch Gesundheitsschutz 50:533-546

45. Kamtsiuris $P$, Lange $M$, Schaffrath Rosario A (2007) Der Kinder- und Jugendgesundheitssurvey (KiGGS): Stichprobendesign, Response und Nonresponse-Analyse. Bundesgesundheitsbl Gesundheitsforsch Gesundheitsschutz 50:547-556

46. Hölling $H$, Kamtsiuris $P$, Lange M et al. (2007) Der Kinder- und Jugendgesundheitssurvey (KiGGS): Studienmanagement und Durchführung der Feldarbeit. Bundesgesundheitsbl Gesundheitsforsch Gesundheitsschutz 50:557-566

47. Filipiak-Pittroff B, Wölke G (2007) Externe Qualitätssicherung im Kinder- und Jugendgesundheitssurvey (KiGGS). Vorgehensweise und Ergebnisse. Bundesgesundheitsbl Gesundheitsforsch Gesundheitsschutz 50:573-577 
48. Dölle R, Schaffrath Rosario A, Stolzenberg H (2007) Der Kinder- und Jugendgesundheitssurvey (KiGGS): Datenmanagement. Bundesgesundheitsbl Gesundheitsforsch Gesundheitsschutz 50 : 567-572

49. Lange M, Kamtsiuris P, Lange C et al. (2007) Messung soziodemographischer Merkmale im Kinderund Jugendgesundheitssurvey (KiGGS) und ihre Bedeutung am Beispiel der Einschätzung des allgemeinen Gesundheitszustands. Bundesgesundheitsbl Gesundheitsforsch Gesundheitsschutz 50:578-589

50. Schenk L, Ellert U, Neuhauser H (2007) Kinder und Jugendliche mit Migrationshintergrund in Deutschland. Methodische Aspekte im Kinderund Jugendgesundheitssurvey (KiGGS). Bundesgesundheitsbl Gesundheitsforsch Gesundheitsschutz 50:590-599

51. Bergmann RL, Bergmann KE, Kollmann F et al. (1977) Wachstum. Atlas. Papillon, Wiesbaden

52. Tanner JM, Whitehouse RH (1976) Clinical longitudinal standards for height, weight, height velocity, weight velocity, and stages of puberty. Arch Dis Childh 51:170-179

53. Danker-Hopfer, Ostersehlt D (1990) Probleme bei der Erfassung und Auswertung von Menarchedaten, aufgezeigt am Beispiel empirischer Daten aus zwei Bremerhavener Wachstumsstudien. Ärztl Jugendkd 81:296-401

54. Schmitz KE, Hovell MF, Nichols JF et al. (2004) A validation study of early adolescents' pubertal selfassesments. J Early Adolescence 24:357-384
Ursula Schlipköter, Manfred Wildner (Hrsg.)

\section{Lehrbuch}

\section{Infektionsepidemiologie}

Bern: Hans Huber 2006, 275 S.

(ISBN 13: 978-3-456), 29.00 EUR

Die Infektionsepidemiologie (IE) hat in

Deutschland in den letzten Jahren einen deutlichen Aufschwung erlebt, institutionell, methodisch, nicht zuletzt auch in der öffentlichen Wahrnehmung. Einem also zunehmenden Bedarf an infektionsepidemiologischer Kompetenz standen bislang erstaunlicherweise kaum deutschsprachige Lehrbücher gegenüber. Das neu vorgelegte Buch stößt in diese Lücke vor.

Das Vorwort verspricht eine "systematische Einführung" in das Thema mit einer "lebendigen Verbindung zur Praxis". Diese Systematik bietet sechs Hauptabschnitte: 1) Grundlagen, 2) Spezielle Methoden, 3) Deskriptive IE, 4) Investigative / Analytische IE, 5) Interventionelle IE und 6) Angewandte IE. Diese Gliederung anhand der Überschriften ist an sich schlüssig, weist aber einige Inkonsistenzen bezügl. der Anordnung der jeweiligen Unterkapitel auf, so hätte man z.B. das Kap. 8„Wirksamkeit von Impfstoffen“ mit Kap. 17 "Immunprophylaxe" in einem Abschnitt zusammenfassen können; es gäbe noch weitere Beispiele. Auch sind die Überschriften z.T. diskutabel, so fällt es doch etwas schwer, Bioterrorismus unter "Angewandte IE" zu subsumieren. Auf jeden Fall wird eine Vielzahl von wichtigen Themen der IE angesprochen, von Standardthemen bis zur modernen "Genetische Epidemiologie"; die getroffene Auswahl kann durchaus als gelungen angesehen werden.

Am z.T. recht unterschiedlichen inneren Aufbau der einzelnen Unterkapitel merkt man dem Buch doch an, dass es von ca. 30 verschiedenen Autoren verfasst wurde. Auch sind die in den jeweiligen Kapiteln angehängten Literaturangaben recht inhomogen, von einer Handvoll Quellen bis zu mehreren Seiten, und sie umfassen insgesamt etwas zu wenige Internetquellen. All das kann man aber sicher in einer Folgeauflage leicht abstellen. Dann sollte man auch ein Glossar einfügen.

Das Buch ist jedoch sehr lesbar geschrieben und hat ein gefälliges Layout, allerdings hätten einige Grafiken mehr noch zusätzlich zur Lesefreude und zum Verständnis beigetragen. Die Autoren sind engagiert bei der
Sache, der dabei an manchen Stellen auftretende Überschwang in den Formulierungen ist da verzeihlich. Ebenfalls bei einer Erstauflage noch verzeihlich sind eine - allerdings nicht ganz geringe - Reihe von kleineren Ungenauigkeiten im Text, z.B. SARS = Sudden Respiratory Distress Syndrome ? Insgesamt bietet das Lehrbuch eine gute Grundlage zum Interesse und Verständnis am Gebiet, und den Autoren bzw. Herausgebern dürfte es gelingen, die kleinen Unzulänglichkeiten zu eliminieren und die vorhandenen Qualitäten des Buches weiter zu stärken.

Priv.-Doz. Dr. med. Dr. PH Reinhard Bornemann

Reinhard Bornemann (Bielefeld) 